Meta

Journal des traducteurs

Translators' Journal

\title{
Interview et interprétation consécutive dans le film Shoah, de Claude Lanzmann
}

\section{Francine Kaufmann}

Volume 38, numéro 4, décembre 1993

Le Je du traducteur

The $I$ of the Translator

URI : https://id.erudit.org/iderudit/003290ar

DOI : https://doi.org/10.7202/003290ar

Aller au sommaire du numéro

Éditeur(s)

Les Presses de l'Université de Montréal

ISSN

0026-0452 (imprimé)

Découvrir la revue

Citer cet article

Kaufmann, F. (1993). Interview et interprétation consécutive dans le film

Shoah, de Claude Lanzmann. Meta, 38(4), 664-673.

https://doi.org/10.7202/003290ar d'utilisation que vous pouvez consulter en ligne.

https://apropos.erudit.org/fr/usagers/politique-dutilisation/ 


\section{INTERVIEW ET INTERPRÉTATION CONSÉCUTIVE DANS LE FILM SHOAH, DE CLAUDE LANZMANN}

FRANCINE KAUFMANN

Université Bar-Ilan, Ramat-Gan, Israël

\section{IMPRESSIONS DE TOURNAGE}

Un matin de septembre 1979, une collègue de la section française de Kol Israël (la radio israélienne) me téléphona pour me dire que Claude Lanzmann cherchait une interprète de l'hébreu au français pour un film sur la Shoah. Elle-même avait été pressentie, après d'autres, mais n'avait pas fait l'affaire. Elle m'avait donc recommandée à Lanzmann en lui disant que non seulement j'appartenais au métier de la radio et de la télévision, mais que, fille de déporté, j'avais fait un doctorat sur la littérature de la Shoah et que, de surcroît, $\mathrm{j}$ 'enseignais et pratiquais l'interprétation de conférence, simultanée et consécutive.

Ce dernier titre suscita plutôt la méfiance de Lanzmann. Dès notre première entrevue, il me déclara qu'il ne voulait surtout pas de ce qu'il appelait «le ton ONU», le débit monocorde et ennuyé des interprètes professionnels qu'il avait entendus à New York. Il souhaitait une interprétation qui ne soit pas mécanique, puisqu'il comptait s'en servir comme partie intégrante de la bande-son, lors du montage définitif. Cette intention m'étonna encore plus lorsque je découvris que le tournage se déroulait à une caméra, que l'interprète restait hors-champ et que, durant la phase d'interprétation en voix off, l'œil de l'objectif restait braqué sur le visage du témoin, réduit momentanément au silence en attendant le fin de la traduction. Je ne voyais pas l'intérêt de ce procédé lourd et fastidieux et j'imaginais mal comment Lanzmann utiliserait au montage ce témoignage à deux temps qui ralentissait le déroulement d'interviews déjà interminables, puisqu'elles se prolongeaient durant de longues heures (avec en moyenne deux jours de tournage par témoignage).

Habituée à ce que l'interprète conseille son «client» sur les modalités pratiques les plus appropriées à son cas, j'essayais naïvement (et en vain) de discuter avec Lanzmann, de lui proposer une interprétation «simultanée chuchotée» (au micro et à l'oreille de l'intervieweur), avec un second micro enregistrant le témoignage en hébreu. Je me disais que - entre autres avantages - je serais préservée ainsi du risque d'oublier un détail ou un mot important, d'autant que Lanzmann exigeait une interprétation consécutive sans «prise de notes». Il voulait que le témoin sente face à lui un traducteur-interlocuteur et non pas un traducteur-technicien. Il était donc convenu que je regarderais le témoin dans les yeux et que j'éviterais de l'interrompre avant qu'il ne s'arrête de lui-même pour me laisser traduire. Il me faudrait donc mémoriser mentalement tout en écoutant. La méthode me semblait périlleuse, voire impraticable pour les longues plages de récit. Mais je crois bien qu'en mon for intérieur je me rassurais en me disant qu'en dernier ressort Lanzmann renoncerait à son projet en visionnant les rushes. C'est que je partais du principe que, si Lanzmann recourait à un interprète, c'est parce qu'il ne comprenait pas la langue des témoins et ne pouvait les interviewer que par l'intermédiaire de cette béquille technique. Une fois le tournage achevé, la béquille n'avait plus (à mes yeux) de raison d'être et pouvait être retirée. Ma logique me soufflait que Lanzmann pourrait alors se résoudre au soustitrage, voire refaire au mixage une traduction en voix off, précise et coulée des seules 
séquences retenues au montage. C'est du moins dans cet état d'esprit que j'abordais le tournage.

Je me trompais, bien sûr. Mais je dois dire à ma décharge qu'à aucun moment Lanzmann ne m'expliqua pourquoi il avait choisi une interprétation consécutive enregistrée sur la bande-son. Il se contenta de m'indiquer comment il voulait que je procède. Il ne m'apprit rien non plus sur la forme et le contenu du film qu'il avait en tête. Il m'expliqua seulement qu'il ne se contentait pas d'enregistrer des témoignages, mais qu'il aspirait à créer LE film qu'on n'avait pas encore réalisé sur la Shoah. Un film de plusieurs heures, avec une version courte pour le cinéma et une version longue diffusée à la télévision en six parties d'une heure. (C'était du moins son projet à l'époque, si ma mémoire ne me trompe pas.) Il avait d'abord eu l'intention d'intituler son œuvre Holocauste, mais la diffusion mondiale de la série américaine de NBC, en 1978, lui imposait de chercher un autre titre. Il fulminait. Mais sa passion et sa conviction étaient communicatives. J'avais vu Pourquoi Isräel?, et je crus aussitôt à la grandeur de son entreprise. D'ailleurs, même si l'équipe avec laquelle il tournait était à bout de forces et de nerfs après avoir parcouru la Pologne, l'Allemagne, les USA... on sentait que chacun participait à une tâche sacrée. L'assistante de Lanzmann, Corinna Coulmass, était moitié allemande et avait fait un doctorat sur la Shoah. Son caméraman était le fils d'un déporté. Nous partagions donc tous la même obsession.

Mon premier jour de collaboration effective fut une expérience traumatisante. Nous avions rendez-vous, dans la forêt de Ben-Chemen, non loin de Jérusalem, avec deux témoins des atrocités de Ponari : Motke Zaïdel et Its'hak Dugin. Je ne connaissais rien de leur histoire. Je n'avais aucune idée du type de témoignage que je devais interpréter. Je ne comprenais pas la réserve, transformée bien vite en franche hostilité, de ces deux Israéliens, pour moi anonymes, vis-à-vis de leur intervieweur. Je ne me rendis compte que beaucoup plus tard, durant le mois de tournage auquel je participais, que Lanzmann avait déjà longuement rencontré tous les témoins, qu'il les avait enregistrés au magnétophone et avait transcrit leur récit, qu'il tentait de leur faire réexprimer à l'écran, avec le maximum d'émotion, de vérité et d'intensité, certaines phrases, certains détails, certaines remarques qu'ils avaient prononcés spontanément, deux ou trois années auparavant, et qui lui paraissaient essentiels pour la progression du film que déjà, visiblement, il portait dans sa tête.

C'était donc une belle journée de fin d'été. Les forestiers de Ben-Chemen avaient mis le feu aux broussailles et l'on voyait monter des flammes derrière le rideau d'arbres et une fumée épaisse qui barbouillait l'horizon. Lanzmann me dit, comme en passant, que la séquence que nous allions tourner devait se raccorder avec une séquence tournée en Pologne. Il tenait beaucoup à ce que nous mettions la double interview en boîte au plus vite, tant que la lourde fumée filtrait à travers les arbres. Malheureusement, de nombreux passages d'avion qui couvraient le son, et des problèmes techniques de caméra perturbèrent l'enregistrement et nous firent prendre un retard considérable. Avec le temps qui passait et la fatigue qui s'installait, les questions précises et crues de Lanzmann révoltaient de plus en plus les témoins qui voulaient nous planter là et rentrer chez eux.

Bien que nouvelle venue dans l'équipe, j'étais la seule à pouvoir m'entretenir avec eux dans leur langue. J'essayais de les convaincre - au nom du directeur - d'aller jusqu'au bout de leur témoignage et d'avoir encore un peu de patience malgré toutes les interruptions techniques. Déchirée entre ma compassion pour les témoins et mon adhésion totale aux objectifs ultimes de Lanzmann, ma position d'interprète devenait de plus en plus intolérable.

D'autant plus que les premières minutes d'enregistrement avaient été particulièrement inhibantes. Coincée contre la caméra (Claude Lanzmann s'était placé de l'autre côté, également hors-champ), je devais interpréter à la fois les questions de Lanzmann du français à l'hébreu et les réponses des témoins de l'hébreu au français. Ce qui, pour certaines interventions alternées, très rapides, des trois protagonistes (ex: Shoah:26) me plaçait dans 
une situation fausse, car je ne pouvais manifestement pas changer de voix pour interpréter tour à tour Lanzmann, Motke Zaïdel ou Its'hak Dugin. Sans compter les problèmes de traduction de A en B (comme disent les interprètes), c'est-à-dire de la langue maternelle (le français pour moi) vers la langue seconde (l'hébreu). C'est ainsi que la toute première question de Lanzmann fut, si ma mémoire est bonne: «Demandez-leur s'il y avait les mêmes bouleaux dans la forêt de Ponari ?» Interloquée, je creusais en vain ma mémoire pour retrouver le mot hébraïque qui signifie bouleau. Bredouille, habituée aux faux départ, à la radio ou à la télévision, je fis signe à l'équipe: «Stop, dis-je, je ne me souviens pas du terme hébraique pour bouleau. Est-ce important?» La réaction de Lanzmann fut sans équivoque.

Mon métier était de connaitre les mots ! Avec des bobines de film de dix minutes et compte tenu du temps perdu pour le cadrage et le clap, je n'allais tout de même pas interrompre le tournage et distraire le témoin chaque fois que se poserait une question de vocabulaire.

Je me le tins désormais pour dit et j'en conclus, peut-être à tort, que si le mot exact n'était pas l'essentiel, l'essentiel était sans doute ailleurs, que le choix des mots ou de leurs équivalences devait être au service de la remémoration et respecter le flot montant de souvenir, tantôt heurté, hésitant, tantôt accéléré. Je renonçais donc mentalement à recourir à une pratique admise en interprétation consécutive et courante dans les médias (même s'il faut n'y recourir qu'exceptionnellement): la possibilité d'interrompre la parole pour procéder à une vérification, demander un rappel ou une explication, ceci pour garantir l'exactitude et la fidélité de cette parole. Je me rassurais intérieurement en me disant que s'il se produisait malgré moi des imprécisions, voire des oublis ou des erreurs dans ma traduction, il resterait une certaine marge de manœuvre au montage et au mixage, et qu'après tout ce n'était pas mon problème mais celui de Lanzmann. Puisqu'il exigeait de son interprète de faire de la haute voltige sans filet, c'est qu'il avait des priorités que j'ignorais, car lui seul disposait d'une vision d'ensemble de l'œuvre à réaliser, de l'objectif à atteindre.

Je remplaçais le mot litigieux «bouleau» par le terme générique: «Est-ce que les arbres d'ici ressemblent à ceux de Ponari ?»

Je reçus comme un coup de poing dans l'estomac l'horrible récit des deux hommes qui revivaient leur travail à mains nues dans les fosses communes dont ils devaient ressortir, pour les brûler, des cadavres plats comme des galettes, parmi lesquels Motke Zaïdel avait reconnu sa propre mère et ses trois sœurs.

Je me souviens de deux ou trois moments où je fus si bouleversée que je cessais de mémoriser pour écouter avec horreur ce récit inhumain. Imperturbable, Lanzmann demandait des détails sur la dimension et la disposition des fosses. Les témoins avaient déjà répondu, mais j'avais oublié de traduire. Lanzmann insistait: «Quelle était la profondeur exacte des fosses?» Prise en flagrant délit d'amnésie, mais seule à m'en rendre compte, je posais à nouveau la même question, navrée d'imposer au témoin, étonné et lassé, cet effort inutile.

Si je rends compte aujourd'hui de tout ce qui m'agitait alors, c'est parce que je me suis très souvent interrogée sur le rôle réel que Claude Lanzmann avait assigné à l'interprétation enregistrée dans la bande sonore du film. Durant la phase du tournage, j'étais consciente d'être placée dans une situation limite, qui ne ressemblait à aucun des cas de figures enseignés dans les écoles. Chaque séquence me laissait frustrée et désorientée, incapable d'utiliser des critères connus pour juger si $\mathrm{j}$ 'avais ou non fait ce que le réalisateur attendait vraiment de moi.

Aujourd'hui que le film est consideré comme un classique, que le texte des soustitres et des séquences interprétées est devenu (à ma grande surprise) un livre traduit dans toutes les langues, que des psychiatres analysent les témoignages recueillis par Lanzmann à travers le discours indirect, l'écho amplifié, déformé ou atténué de la traduction, langage 
canalisé encore, et émondé par le montage, je crois utile de tenter d'apprécier la fonction cinématographique de l'interprétation consécutive dans le film Shoah.

\section{LES LANGUES DE SHOAH}

Pour préparer cet article, j'ai relu le livre réunissant les textes français du film, que je citerai dans l'édition de poche, ainsi que l'excellent recueil d'articles, d'analyses et d'interviews publiés sous le titre $A u$ sujet de Shoah. Dans ce dernier livre, je me suis plus particulièrement arrêtée sur les analyses de Shoshana Felman ( $c f$. le paragraphe «La multiplicité des langages»: 64-65) et d'Anny Dayan-Rosenman ( $c f$. le sous-chapitre : La traduction: la voix du traducteur: 192-194) et sur les interviews de Lanzmann publiées dans Les cahiers du cinéma (1985) et dans La nouvelle revue de psychanalyse (1986) (ibid: 280 à 305).

J'ai aussi rencontré à Herzlia l'autre traductrice israélienne du film, Fanny Apfelbaum, qui a traduit du yiddish le témoignage de Mordekhaï Podchlebnik (Shoah: 22, 25, 104-105). Et j'ai revu en vidéo, décrypté, chronométré et analysé la bande-son et le texte publié des témoignages que j'ai personnellement traduits de l'hébreu (Its'hak Dugin, Motke Zaïdel et sa fille 'Hanna: 22, 23, 26 à 29; Antek [It'shak Zuckermann] et Kajik [Sim'ha Rotem]: 242 à 249).

Le développement qui va suivre n'est qu'un bref condensé de mes conclusions personnelles, fondées souvent sur des observations relevées dans Au sujet de Shoah.

En ce qui concerne les langues du film, elles sont au nombre de six : quatre européennes (le français, l'anglais, l'allemand, le polonais, sans compter quelques bribes de grec et de lithuanien) et deux juives (l'hébreu et le yiddish).

Force nous est de constater que la seule langue qui fasse foi (pour reprendre un concept juridique), celle dans laquelle le film (puis le livre du film) est pensé, conçu et réalisé est le français: langue de l'enquêteur-réalisateur «qui n'est celle d'aucun témoin (ce qui ne peut pas être un hasard)» remarque Shoashana Felman (op.cit. : 65). (Elle oublie, à juste titre, le français approximatif du survivant de Corfou, un français écorché qui, à l'évidence, n'est pas l'une de ses langues d'expression courante: 163 à 166.)

Les autres langues sont, soit sous-titrées ultérieurement en français à l'intention du spectateur, lorsque Lanzmann les comprend en direct (c'est le cas pour l'allemand et l'anglais), soit traduites en français par une interprète (le polonais, le yiddish et l'hébreu). La nécessité ou non de traduire en français dépend, par conséquent, d'une banale contrainte technique conditionnée par le bagage linguistique du réalisateur, premier auditeur, premier destinataire des messages verbaux recueillis dans ce film, mais aussi premier témoin parti à la recherche d'une réalité en train de mourir une seconde fois, avec l'inéluctable disparition prochaine des derniers témoins oculaires de la Shoah, et qui invite le spectateur à le suivre dans sa quête.

À un second degré, la multiciplicité des langues étrangères dans Shoah, et donc la nécessité de la traduction pour permettre la communication, reflète l'un des aspects spécifiques des crimes nazis: l'ampleur de leur expansion spatiale et idéologique à travers l'Europe et une partie de l'Afrique, avec pour conséquence l'éclatement et l'annihilation des centres de peuplement d'origine et la dispersion planétaire des témoins après le cataclysme (Lanzmann a tourné dans 14 pays). L'acclimatisation des rescapés dans un nouvel environnement a exigé d'eux une réadaptation linguistique. C'est ainsi qu'aucun des rescapés, parlant anglais ou hébreu dans le film, ne s'exprime dans sa langue maternelle. Un seul semble avoir exigé de témoigner dans sa langue d'origine: Mordekhaï Podchlebnik. Fanny Apfelbaum se souvient en effet qu'il parlait correctement 1'hébreu avec sa famille. Mais le choix délibéré du yiddish est significatif. N'a-t-il pas confié à sa traductrice n'avoir jamais raconté son histoire à ses enfants parce que ceux-ci ignorent le yiddish: «Ces choses-là, on ne peut les dire qu'en yiddish», a-t-il expliqué à Fanny. 
Pour les autres, il n'est pas impensable, au contraire, que l'extranéité linguistique les ait délivrés d'un tabou lié à l'impossibilité du témoignage parce que, d'une certaine manière, la réalité de la Shoah est incommunicable.

Dans un colloque sur la Shoah, organisé à Paris en 1988, je notais un phénomène similaire dans la littérature de l'après-guerre. Les écrivains juifs consacrés n'ont pas su ou pas pu, semble-t-il, réagir littérairement à l'événement. Par contre, on a vu surgir une pléiade d'écrivains dont la langue maternelle n'était pas le français et qui publièrent, dans les annés 50 et 60 , les premiers chefs-d'œuvre de la littérature française de la Shoah: Elie Wiesel, André Schwarz-Bart, Romain Gary, Manès Sperber, Anna Langfus, Piotr Rawicz, Wladimir Rabi... Tout se passe comme si, constatais-je alors, «le maniement d'une langue étrangère pour traduire les réalités autres de la planète Shoah leur permet de vaincre plus facilement l'obstacle qu'il y a à dire l'inhumain avec des mots humains. Car les connotations, si familières et rassurantes d'une langue maternelle, peuvent devenir encombrantes pour exprimer le scandale de la Shoah. Mais quiconque utilise une langue apprise sur le tard peut la disloquer et la manipuler plus aisément... Les écrivains d'Europe de l'Est purent vaincre l'interdit qui pesait sur une expression esthétique de la Shoah en la traduisant en langue étrangère. La seule qui convienne à un événement indicible (Penser Auschwitz: 69-70, cf. Bibliographie).»

Dans une certaine mesure, les témoins, dans le film Shoah, bien que n'étant pas soumis dans leur témoignage à une contrainte esthétique, sont conscients de la difficulté d'organiser leurs souvenirs pour les transformer en mémoire transmise. Puis, de traduire en paroles cohérentes, articulées et compréhensibles pour le spectateur, une réalité inhumaine dans laquelle le langage lui-même participait à l'entreprise de déshumanisation des victimes: «Les Allemands nous imposaient de dire, concernant les corps, qu'il s'agissait de Figuren, c'est-à-dire de... marionnettes, de poupées, ou de schmattes, c'est-à-dire de chiffons» (Shoah: 26).

Lorsque le courrier polonais Jan Karsky est contacté, en 1942, par deux leaders juifs qui lui demandent de pénétrer physiquement dans le ghetto de Varsovie pour témoigner de ce qui s'y passe («Nous sommes humains, comprenez-vous?», lui disent-ils, (Shoah: 211)). Mais lui se souvient:

Ce n'était pas un monde.

Ce n'était pas l'humanité. [...]

On me disait qu'ils étaient des êtres humains.

Mais ils ne ressemblaient pas à des être humains

(Shoah : 221)

\section{LA FONCTION MÉTAPHORIQUE DE LA TRADUCTION}

À ce troisième degré, la traduction dans le film Shoah remplit une fonction métaphorique, comme l'a finement noté Anny Dayan-Rosenman:

La traduction apparaît comme l'une des figures majeures de toute l'entreprise: la nécessité de traduire, l'urgence de transmettre et, au cœur même de ce travail, la certitude qu'il existe un noyau irréductible à toute traduction, à toute transmission.

Traduire, c'est toujours passer d'un monde à l'autre. Mais quand il s'agit de Treblinka ou de Chelmo? [...] En ce sens s'organise, sous nos yeux, une métaphore de tout témoignage. Car la distance qui sépare la parole dite de la parole traduite n'est qu'une faible mesure de celle qui sépare le vécu du souvenir et le souvenir de la parole (Au sujet de Shoah : 192-193).

Il faut noter que la fonction métaphorique de la traduction renvoie non seulement au point de vue du témoin, mais aussi à celui de l'enquêteur (Claude Lanzmann) et du spectateur du film. L'analyse de Shoshana Felman est à cet égard très éclairante : 
L'hétérogénéité des positions cognitives de vision ou de non-vision, sont amplifiées et redoublées dans le film par la pluralité des langages dans lesquels nous sont proposés les témoignages [...] L'étrangeté palpable des langues dans le film est emblématique de l'étrangeté radicale de l'expérience de l'Holocauste, pas seulement pour nous, mais aussi pour ses protagonistes [...] C'est une métaphore du film que sa langue soit une langue de traduction, et, en tant que telle, doublement étrangère : que l'événement advienne dans des langues étrangères à celle du film, mais aussi que la signification de l'événement ne puisse s'articuler que dans une langue étrangère aux langues de l'événement (le français) (ibid: 64-65).

Cette fonction métaphorique de la traduction est figurée par un procédé cinématographique que Shoshana Felman appelle : L'effet de retard dû à :

L'incapacité du regard de voir et de traduire simultanément en sens [...] Le film nous place dans la situation du témoin qui voit et entend, mais ne peut pas comprendre ce qui se passe jusqu'à la dernière intervention quand l'effet de retard prend fin et quand la signification de l'information visuelle / acoustique est délivrée par l'interprète, qui, elle-même, d'une certaine manière, le gauchit et lui fait écran (ibid: 64 ).

Ainsi, même les inexactitudes, les infidélités, les erreurs et les omissions de la traduction sont appelées à jouer un rôle dans le film et c'est pourquoi elles sont conservées au tournage. L'infidélité de la traduction est métaphore de l'infidélité de la mémoire et de l'infidélité de l'écoute, même attentive, d'un observateur qui ne peut rester qu'extérieur à la planète Shoah. Tout ce qui, durant le tournage même de Shoah me préoccupait, m'inquiétait, me semblait un obstacle encombrant à la communication et à la transmission fidèle de l'information résultait d'un procédé cinématographique délibéré : même ce que j'appelais le témoignage à deux temps, le récit en langue originale suivi de sa traduction enregistrée sur la bande sonore.

En regardant attentivement certaines séquences, $\mathrm{j}$ 'ai d'ailleurs constaté que Lanzmann et Ziva Postek (l'admirable monteuse du film) avait souvent couvert le témoignage original par les images des lieux évoqués, lieux réels mais vides de toute action vraie: seule la parole des témoins y rend présente la Shoah. Et quand vient le moment de l'interprétation en français, l'écran nous montre le visage du témoin qui semble écouter l'écho de sa propre mémoire, déformée et altérée par la double distance de la langue étrangère et de la subjectivité de l'interprète (résigné peut-être par avance à n'être pas vraiment entendu mais seulement écouté?).

Cet effet de décalage qui permet en définitive une identification du spectateur avec l'auteur du film (l'enquêteur) est bien décrit par Dan Fainaru, critique de cinéma au Jérusalem Post Magázine, qui s'exaspère tout d'abord de la maladresse apparente de l'encombrant système de traduction, alors qu'il aurait été si naturel de recourir à la voix off:

But after a few minutes the reason for this becomes evident. In this fashion, a certain rythm is imposed on the film. You soon realize there is a certain degree of identification with the person asking the questions, namely Lanzmann, and like him, you need the spare time of the translations, to have the facts sink in fully and leave their full impact. And one can't help but marvel at the accuracy of the tone, at the authenticity of the feelings emerging from the soundtrack (Jerusalem Post, 12/06/1986).

Si Shoah était un banal documentaire, il n'y aurait sans doute pas lieu de s'étonner du ton juste, de l'authenticité des sentiments qui se dégagent de la bande sonore. Il en va tout autrement si Shoah est une fiction comme l'indique clairement la première phrase du film, inscrite sur l'écran alors que la bande-son reste résolument muette : «L'action commence de nos jours à Chelmo-sur-mer, Pologne» (Shoah: 17). 


\section{LA TRADUCTION, FICTION DU RÉEL}

La question qui se pose alors est pourquoi, si Lanzmann avait sans doute conçu très tôt la traduction comme métaphore de l'incommunicabilité de l'expérience-Shoah (puisqu'il avait inclus, dès l'origine, une part d'interprétation dans le corps du film, de manière indissoluble, intrinsèque), pourquoi donc s'est-il soigneusement abstenu d'expliquer ses objectifs à ses interprètes?

La seule explication logique, à mes yeux, est que Lanzmann voulait mettre en scène ses traductrices comme il a mis en scène ses témoins.

En ce qui concerne ses témoins, Lanzmann s'est longuement expliqué dans des interviews et lors de colloques. Pour Les cahiers du cinéma, il retrace le processus qui l'a amené à faire de Shoah «non pas un documentaire» (Au sujet de Shoah: 298), «non pas un film historique» (ibid: 303), «non pas un film d'archives» (ibid: 297), mais «un film de vie, avec du présent pur» (ibid: 297), à partir d'une mise en scène qui transforme les témoins en acteurs et le film en «fiction du réel» (ibid: 301 ). Non pas une «reconstitution», mais une «démonstration» où le protagoniste retrouve spontanément au présent les gestes qu'il accomplissait dans ce passé-là (ibid: 289). «C'est leur propre histoire qu'ils racontent. Mais la raconter ne suffisait pas. Il fallait qu'ils la jouent (ibid: 289).»

C'est pourquoi Claude Lanzmann loue une authentique locomotive polonaise pour pénétrer à Treblinka avec l'ancien conducteur de locomotive qui dirigeait le transport des juifs. C'est pourquoi il loue un salon de coiffure à Tel-Aviv pour faire revivre à Abraham Bomba les moments où il coupait les cheveux aux femmes entrant dans la chambre à gaz (ibid: 297 à 300). Mais pour obtenir que ses témoins revivent ces instants d'extrême intensité devant la caméra, il évite de déflorer les choses (ibid: 299). Il ne leur dit pas à l'avance ce qu'il attend d'eux.

À la lecture de cet interview de Lanzmann, j'ai soudain compris le rôle essentiel du rideau de flammes et de fumée dans la forêt de Ben Chemen, pour mettre en condition Its'hak Dugin et Motke Zaïdel. Et j'ai compris leur impatience et leur hostilité : ils se croyaient là seulement pour raconter.

J'ai compris aussi pourquoi Lanzmann avait demandé à Sim'ha Rotem (Kajik) de quitter Jérusalem à l'aube pour tourner non loin de Haïfa, dans le musée du Kibboutz Lo' hamey Haguettaoth, un kibboutz fondé par les survivants du ghetto de Varsovie. Les retrouvailles émues de Kajik avec son ancien commandant de l'OJC se sont passées horscaméra. Mais la présence d'Antek pendant le témoignage de Kajik est figurée par des gros plans muets d'Antek, insérés au montage (dans la réalité, il est filmé pendant qu'il écoute ma traduction). La maquette de Varsovie que Lanzmann impose à Kajik de décrire du doigt et du regard et de contourner physiquement (processus télescopé au montage) joue un rôle de catalyseur dans la montée du souvenir.

D'ailleurs, après ces premières mises en scène, après ces remémorations extorquées sans préparation aux protagonistes désorientés, Lanzmann termine à loisir le tournage au domicile du témoin quelques jours plus tard. Mais le ton est donné. Et l'hostilité de l'interviewé a eu le temps de s'évanouir.

Comme le témoin, l'interprète est mis en condition, manipulé et mis en scène. Je me croyais là pour traduire. Je voulais prendre des notes, être complète et fidèle. Je ne pouvais me douter que j'étais un protagoniste du film, chargée d'incarner le premier spectateur du film. En effet, Lanzmann connaissait déjà dans le détail le récit dont les rescapés étaient les acteurs. Dans le trilogue qui s'établissait, moi seule ignorais tout du scénario. Moi seule découvrais l'intrigue une fois la caméra enclenchée. Je comprends aujourd'hui pourquoi Lanzmann me cacha d'abord qu'il existait un script dactylographié des entretiens préalables au tournage, puis qu'il refusa de me les communiquer sous un prétexte quelconque (sauf une fois, pour un témoignage particulièrement précis et insou- 
tenable, non retenu dans le film). Il était nécessaire que ma surprise, que mon horreur, que ma compassion surgissent avec le maximum d'intensité et d'authenticité, dans ma voix. Contrairement aux règles les plus élémentaires de ma profession, il fallait donc que je sois aussi peu préparée que possible aux révélations que j'allais traduire. Je me souviens d'ailleurs m'être identifiée totalement à 'Hanna Zaïdel, la fille de Motke, dont il n'était pas prévu que je traduise le témoignage (Shoah: 22-23). Malgré une expérience très différente, je savais que je partageais le même secret de fille d'un revenant de là-bas. Et je retrouve avec étonnement, sur la bande sonore, l'étrange excitation (qui détonne, se juxtaposant à la voix calme de Kajik) que le récit, délibérément détaché, d'un authentique survivant des combattants de Varsovie suscitait en moi. J'éprouvais (en découvrant au fur et à mesure son aventure personnelle) une véritable exaltation à voir s'animer sous mes yeux un fragment d'épopée vivante de l'histoire collective de mon peuple.

Je comprends rétrospectivement certaines colères de Lanzmann lorsque, entre deux prises de vue, je conversais avec les témoins: il m'accusait de les déconcentrer. Je devine aujourd'hui qu'il souhaitait, de surcroît, que je demeure, moi aussi, silencieuse, recueillie, dans l'état d'hébétude et de révolte suscité par le récit de notre interlocuteur. Il m'avait d'ailleurs formellement interdit de m'entretenir avec eux, hors-caméra, de leurs souvenirs de la Shoah. Je devais me contenter de banalités avant ou après le tournage, même lorsque je fus accueillie durant deux jours dans la chambre d'amis d'un témoin (non retenu dans le film). Ma position était pour le moins inconfortable !

\section{TROIS TRADUCTRICES, TROIS VOIX}

Faut-il conclure que les trois traductrices jouent chacune dans le film un rôle similaire qui permet à Shoshana Felman de parler du «personnage de la traductrice» qui «n'est délibérément pas exclu du film - au contraire elle est souvent présente à l'écran aux côtés de Lanzmann, comme les autres acteurs de l'œuvre» (op.cit.: 64). Il est vrai que Shoshana Felman suit en cela les indications de Lanzmann qui, dans l'avant-propos au texte intégral du film (paroles et sous-titres pour reprendre sa définition), affirme : «Les langues que je n'entendais pas sont traduites en français dans le corps même du film, les interprètes étant elles-mêmes présentes à l'image.»

De fait, seule l'interprète polonaise est présente à l'image, aux côtés de Lanzmann. Les interprètes israéliennes sont hors-cadre, comme Lanzmann lui-même. Il semble que lorsque l'enquêteur se promène physiquement avec son interprète sur les lieux mêmes du crime, en Pologne, leur présence matérialisée à l'écran s'impose dans la logique du processus de quête topographique, de localisation dans le paysage, de recherche de traces tangibles dans l'espace. Tandis que pour les interviews réalisées loin de l'Europe, sans ancrage dans les lieux réels du drame, quand le témoignage s'appuie uniquement sur la parole, quand le voyage s'accomplit uniquement dans la mémoire - qui abolit l'espace et le temps — l'enquêteur et l'interprète restent extérieurs à l'image, à l'écoute tendue du pouvoir évocateur des mots, des silences, du regard des rescapés.

Je ne sais pas à quel moment ce procédé s'est imposé à Lanzmann. En tout cas, Israël semble avoir été la dernière étape du tournage. Or, Fanny Apfelbaum se souvient qu'au cours de l'unique journée de tournage auquel elle a participé, en mai 1979, Lanzmann l'avait d'abord placée aux côtés de Mordekhaï Podchlebnick, devant la caméra. Il avait même réalisé des essais de cadrage. C'est Fanny qui a refusé obstinément d'être filmée et a obtenu de rester hors-cadre. Le résultat a sans doute été convaincant, puisqu'en septembre et octobre 1979 Lanzmann avait décidé que lui-même et son interprète ne figureraient pas à l'image.

On peut en conclure que la fonction cinématographique de la traduction (et de l'interview) n'est pas conçue de la même manière selon que l'accent est mis sur le lieu ou 
sur la parole. Mieux, que le personnage de la traductrice change en conséquence. Anny Dayan-Rosenman a bien senti que chaque traductrice a sa particularité. Pour l'hébreu, la voix est juvénile et dit je et nous quand le témoin dit je et nous. La traductrice du yiddish «est en sympathie avec le témoin, mais elle sait qu'elle n'est pas lui, elle dit $i l »$. La traductrice polonaise «garde ses distances, face au témoin, face aux mots, face au récit». «Elle appelle le témoin monsieur.» «Elle a des réticences à traduire certaines réponses et se fait rappeler à l'ordre par Lanzmann» (Au sujet de Shoah: 193-194).

De fait la personnalité de chaque interprète est différente et correspond à une distribution et à une mise en scène différente.

Barbara Jannica, la traductrice polonaise, est en fait une guide-interprète officielle, chargée par la République populaire de Pologne d'accompagner l'équipe de Lanzmann. Ce dernier témoigne des rapports orageux qui régnèrent parfois entre eux. Quand les paysans polonais, devant l'église, parlent des juifs:

Ils disent : Jidki, les youpins; elle traduit en bonne catholique polonaise : Jidi, les juifs [...] Il y a des moment où sa haine de moi était si forte! Elle était très étrange. Parfois elle gauchissait tout, mes questions, les réponses. Elle les adoucissait. À d'autres moments elle était coincée par la vérité et elle explosait dans sa traduction d'une façon très brutale ( $A u$ sujet de Shoah: 284 et 287 ).

De fait Barbara Jannica remplit son rôle à contrecceur. Elle voudrait faire écran à ce qui n'est pas présentable, expliquer la Pologne au visiteur plutôt que de traduire ses questions têtues. Lorsqu'un villageois imite les conversations en yiddish en faisant entendre un ra ra ra, Lanzmann lui lance: «Qu'est-ce qu'il a voulu dire : «la la la », qu'est-ce qu'il essayait d'imiter?"

Et au lieu de se tourner vers le paysan, Barbara répond elle-même: «leur langue» (Shoah: 44).

Parfois elle commente: quand le cheminot de Treblinka éclate en pleurs au souvenir d'une mère assassinée d'une balle en plein cœur, elle s'attendrit: «Monsieur vit ici depuis très longtemps, on ne peut pas l'oublier» (ibid: 43).

Mais lorsqu'elle dit «Monsieur se souvient que... Madame pense que...», il faut reconnaître que Lanzmann entretient délibérément la distance dans ses questions: «Barbara, dis à monsieur et à madame qu'ils habitent une très belle maison. Ils sont d'accord? Est-ce qu'ils la trouvent belle cette maison? » (ibid: 110).

En somme, puisque Barbara Jannica est et se comporte comme une guide-interprète, Lanzmann l'utilise en tant que telle.

Il en va tout autrement de Fanny Apfelbaum. Lanzmann l'a contactée en Israël, parce qu'il l'avait connue dans les salons parisiens et savait qu'elle parlait yiddish, qu'elle avait connu de près la Shoah, que son mari était un rescapé du ghetto de Varsovie. Cultivée, dotée d'une forte personnalité et d'une grande sensibilité, elle ne possédait aucune formation ni aucune expérience de traductrice et se souvient avoir essayé de raconter ce qu'elle entendait sans trop se poser de questions sur les procédés employés. Bouleversée par la manière dont Lanzmann avait brisé son témoin (on se souvient de sa question brutale: «Pourquoi est-ce qu'il sourit tout le temps?» (Shoah:22)), elle s'implique personnellement, n'hésite pas à interroger elle-même, à compléter les questions suspendues de Lanzmann. Et si, malgré son empathie, elle dit $i l$ en parlant du témoin, c'est sans doute en partie parce que Lanzmann emploie toujours le langage indirect: «Qu'est-ce qui est mort en lui à Chelmo?» «Est-ce qu'il trouve que c'est bien d'en parler?» (ibid: 22)

La dernière et troisième interprète appartient à la génération des enfants de rescapés et c'est la seule interprète professionnelle, formée aux techniques de l'interprétation de conférence. 
Ainsi, lorsque Lanzmann interroge 'Hanna Zaïdel par l'intermédiaire de l'interprète: «Pourquoi a-t-elle été tellement curieuse de cette histoire ?» L'interprète, habituée à se faire le porte-parole du locuteur, et non à rapporter la teneur de ses propos à la troisième personne, emploie naturellement le «Je»: «Je sais que lorsque j'étais une toute petite fille, j'avais très peu de contacts avec mon père (ibid: 22 ).» Il s'agit là autant d'une technique apprise et longtemps pratiquée que d'une identification (réelle) avec le sujet interprété.

L'interprète professionnelle est la seule à résister à la tentation de couper le témoin pour traduire avant d'oublier. Mais a-t-elle raison? Si l'effort d'écoute et de mémorisation favorise la concentration des témoins (comme Lanzmann le souhaitait), le rythme du film n'en est-il pas ralenti?

Ainsi le témoignage de 'Hanna Zaïdel dure-t-il 42 secondes, sans la moindre interruption et a été presque intégralement reconstitué en un temps égal : 42 secondes ! Mais dans le témoignage de Sim'ha Rotem, il y a une première plage de 56 secondes sans interruption, traduite en 41 secondes, en oubliant la dernière phrase. Quant à la dernière intervention, si souvent citée, celle qui clôt le film, elle dure 51 secondes. Au bout de 23 secondes, l'interprète tente de commencer à traduire. Mais concentré sur son récit, Kajik ne se laisse pas interrompre. Ici la traduction est condensée et il manque des éléments d'autant plus importants qu'il s'agit des dernières phrases du film. Voici, littéralement, la seconde partie de la dernière intervention de Kajik (en 26 secondes) :

Alors, lorsque je m'en suis retourné dans le ghetto, à un certain moment, j'ai ressenti un calme complet, une sérénité de l'âme. Je pensais que j'étais le dernier homme, le dernier juif, et je n'avais plus du tout envie de repartir dans les égouts. J'ai décidé de rester et d'attendre jusqu'au matin les Allemands. Et qu'alors... ce serait la fin.

Le dernier mot du film est donc (en hébreu) le mot : «fin»». Mais alors vient la traduction :

Et je me souviens d'un moment où j'ai ressenti une espèce de sćrénité, où je me suis dit: «Je suis le dernier juif, je vais attendre le matin et je vais attendre les Allemands (Shoah: 249).»

Il est probable qu'une prise de notes aurait permis d'éviter les omissions. Mais j'ai constaté avec stupéfaction qu'un critique avait reconstitué, malgré l'ellipse, la totalité du sens exprimé : «Au fond c'est le dernier homme disant: je ne me cache plus, je veux mourir avec l'aube.» (Sami Naïr, Au sujet de Shoah: 168) C'est au spectateur, au cinéma, de mesurer les pertes et les gains réels du procédé de traduction choisi par Lanzmann. Mais puisque le montage conserve, juxtaposées, l'originale et sa reformulation par l'interprète et joue du décalage ou de l'adéquation entre les deux, il faut apprécier le contrepoint que constitue le témoignage brut et sa recomposition par le premier spectateur: l'interprète.

\section{RÉFÉRENCES}

LANZMANN, Claude (1985): Shoah, préface de Simone de Beauvoir, Fayard, Le livre de poche $n^{\circ} 6210$, 1986.

Collectif (1990) : Au sujet de Shoah, le film de Claude Lanzmann, Berlin, Coll. «L'extrême contemporain».

GRANCHER, Jacques (Éd.) (1990): Shoah le film: des psychanalystes écrivent, Paris.

D'ALLONES REVAULT, Myriam (1989): «Shoah ou la fiction du réel», Cahiers Bernard Lazare, $\mathrm{n}^{\circ} 125$, pp. 95-109.

DEGUY, Michel (1990) : «Au sujet de Shoah», Cahiers Bernard Lazare, n 126, pp. 103-110.

TRIGANO, Shmuel (Dir.) (1989) : «Penser Auschwitz», Pardès, 9-10, numéro spécial, Éd. du Cerf.

MOSKOVITZ, Jean-Jacques (1991): D'où viennent les parents?, coll. «L'Ancien et le nouveau», Paris, Armand Colin (chap. X sur Shoah). 